\title{
Determination of the Complex Moduli of Viscoelastic Isolator Mounts by Finite Element Modeling and Vibration Testing
}

\author{
Stanley G. Hutton ${ }^{(1)}$, Guoping Chen $^{(2)}$ \\ (t) Mechanical Engineering Department, The University of British Columbia, \\ Vancouver, British Columbia, Canada, V6T 1Z4, \\ (2) Institute of Vibration Engineering, Nanjing University of Aeronautics and Astronautics, \\ Nanjing, Jiangsu, China, 210016
}

\begin{abstract}
An investigation aimed at determining the complex moduli for a number of polymeric materials is discussed. The complex moduli are determined over a particular frequency range on the basis of transmissibility measurements conducted on mass loaded cube-like specimens subjected to controlled base harmonic excitation. An equivalent one dimensional viscoelastic model is created by condensation of the governing equations and the use of an elastic finite element calculation. Complex moduli are determined by comparison of the transmissibilities measured and those predicted by the condensed model. Consideration is also given to the manner in which the experimental data may be fitted as an analytic function of frequency and to the errors that are involved.
\end{abstract}

\section{INTRODUCTION}

The function of an isolator is to reduce the magnitude of motion transmitted from a vibrating foundation to an item of equipment, or to reduce the magnitude of the force transmitted from the equipment to its foundation. ${ }^{1,2}$ Polymeric isolators are commonly used in such systems to minimize vibration levels and to reduce the effect of transmitted vibration. ${ }^{3}$ In this type of isolator, both the load-supporting functions and the energy-dissipating functions are performed by the same element. Such polymers are often modeled as hereditary-elastic materials, i.e. materials whose constitutive relationship can be expressed in the form ${ }^{4,5,6}$

$$
\sigma_{j k}(t)=G_{j k l m}(0) \varepsilon_{l m}(t)+\int_{0}^{t} \varepsilon_{l m}(t-\tau) \frac{d G_{j k l m}(\tau)}{d \tau} d \tau=G_{j k l m} * d \varepsilon_{l m},
$$

where $G_{j k l m}(t)$ are known as the relaxation kernels for the material and * is used to denote the Stieltjes convolution. The number of independent relaxation kernels is dependent upon the anisotropic characteristics of the material. For an isotropic medium, there only are two kernels, $G_{1}(t)$ and $G_{2}(t)$

$G_{j k l m}(t)=\frac{1}{3}\left(G_{2}(t)-G_{1}(t)\right) \delta_{j k} \delta_{l m}+\frac{1}{2} G_{1}(t)\left(\delta_{j l} \delta_{k m}+\delta_{j m} \delta_{k l}\right)$,

and the viscoelastic constitutive equations can be written in terms of the deviatoric components of stress $s_{j k}$ and strain $e_{j k}$

with

$$
\begin{gathered}
s_{j k}=G_{1} * d e_{j k}, \\
\sigma_{l l}=G_{2} * d \varepsilon_{l l}
\end{gathered}
$$

$$
\begin{aligned}
s_{j k}=\sigma_{j k}-\frac{1}{3} \delta_{j k} \sigma_{l l} & s_{j j}=0, \\
e_{j k}=\varepsilon_{j k}-\frac{1}{3} \delta_{j k} \varepsilon_{l l} & e_{j j}=0 .
\end{aligned}
$$

If the response of the specimen is harmonic such that $\varepsilon_{j k}(t)=\varepsilon_{j k} e^{i \omega t}$, then

$$
\sigma_{j k}(t)=G_{j k l m}^{*}(i \omega) \varepsilon_{l m} e^{i \omega t},
$$

where $G_{j k l m}^{*}(i \omega)$ are called the complex viscoelastic moduli, and can be expressed in the form

$$
G_{j k l m}^{*}(i \omega)=a_{j k l m}^{(0)}-\sum_{n=1}^{N} \frac{a_{j k l m}^{(n)}}{a_{n}+i \omega}
$$

and there exist the following relations between $G_{1}^{*}(i \omega), G_{2}^{*}(i \omega)$ and complex Young's modulus $E^{*}(i \omega)$, complex Poisson's ratio $v^{*}(i \omega)$

$$
E^{*}(i \omega)=\frac{3 G_{1}^{*}(i \omega) G_{2}^{*}(i \omega)}{G_{1}^{*}(i \omega)+2 G_{2}^{*}(i \omega)} ; \quad v^{*}(i \omega)=\frac{G_{2}^{*}(i \omega)-G_{1}^{*}(i \omega)}{G_{1}^{*}(i \omega)+2 G_{2}^{*}(i \omega)} .
$$

The representation (4) may or may not represent adequately the constitutive characteristics of the material under consideration in the frequency range of interest. More accurate representation may be found, ${ }^{7}$ but the form assumed in this paper has the advantage of leading to closed form solutions for the free vibration response of such materials. ${ }^{8,9}$

\section{MODELING OF THE VIBRATION RESPONSE OF THE TEST SPECIMENS}

In this section the equations of motion describing the vibration response of three dimensional isolator samples is presented. Using a condensed version of these equations, applied to a particular experimental setup, enables the transmissibility characteristics of the samples under test to be represented by a one dimensional viscoelastic rod equation. A simple three dimensional elastic finite element calculation is required to determine the equivalent stiffness of the model. The model may then be used to determine the required complex viscoelastic moduli based upon the experimentally determined transmissibilities. 